\title{
Gyrokinetic Study of the Local Entropy Dynamics in Turbulent Plasmas with Zonal Flow
}

\author{
Kenji IMADERA, Yasuaki KISHIMOTO, Jiquan LI and Takayuki UTSUMI ${ }^{1)}$ \\ Department of Fundamental Energy Science, Graduate School of Energy Science, \\ Kyoto University, Gokasho, Uji, Kyoto 611-0011, JAPAN \\ 1) Department of Electronics and Computer Science, Tokyo University of Science, Yamaguchi, \\ 1-1 Daigaku-dori, Sanyou-Onoda, Yamaguchi 756-0884, JAPAN
}

(Received 9 December 2009 / Accepted 19 March 2010)

\begin{abstract}
An entropy balance equation that maintains the dynamics in the radial direction is derived to study the role of zonal flows in turbulent transport. The equation describes the dynamics not only of the entropy density production related to zonal flow and local heat flux, but also of entropy density convection. We investigated these spatiotemporal dynamics of entropy in ion temperature gradient driven turbulence based on a global gyrokinetic Vlasov simulations in a slab geometry. The contribution of zonal flow to entropy production is observed to be small in the strong instability case, whereas the zonal flow energy is sufficiently large. We also found that the entropy density with $n=2$ in the Hermite polynomial expansion in velocity space plays an important role in convecting the entropy density, causing turbulent spreading.
\end{abstract}

(c) 2010 The Japan Society of Plasma Science and Nuclear Fusion Research

Keywords: entropy balance, heat transport, zonal flow, gyrokinetic

DOI: $10.1585 /$ pfr.5.S2050

\section{Introduction}

Turbulent transport in magnetically confined fusion plasmas exhibits various prominent features characterized by different time and spatial scales. Zonal flows, which are toroidally and poloidally symmetric potential structures and generated nonlinearly from micro-scale turbulence, are found to play an important role, leading to the regulation of turbulence and transport suppression [1]. Zonal pressure, which is the pressure counterpart of zonal flow, causes pressure corrugations in the radial direction at shorter scale lengths and triggers critical gradient transport with intermittent behavior [2]. Furthermore, turbulence has been found to spatially evolve even in linearly stable regions, which is referred to as turbulent spreading, and non-locally transfer energy to a wider radial region [3].

Various theoretical and computational efforts have been made to understand such complex transport behaviors. A statistical approach is one way to identify such turbulent plasmas. The non-diffusive nature of turbulent transport in the presence of zonal flows has been studied using probability density functions $[4,5]$. Alternatively, based on a thermodynamics approach, entropy has been introduced, which has the advantage of connecting micro-scale phase space structure to macro-scale thermodynamic quantities. The entropy balance equation, which investigates the relationship between entropy production due to the generation of turbulence and the resulting heat flux, has been discussed [6]. However, the effect of zonal flows is masked in the conventional entropy balance equation, since zonal flows correspond to density perturbations, which vanish once they are integrated over the entire real space.

Here, we extended the entropy balance equation that maintains the dynamics in the radial direction, so that it can describe the spatio-temporal dynamics not only of the entropy density production related to zonal flow and local heat flux, but also of entropy density convection. We recently developed a global gyrokinetic Vlasov code on the basis of the conservative form of the interpolated differential operator (IDO-CF) scheme, which captures fine scale structure without causing serious numerical phase errors [7]. Using this code, we investigated the entropy dynamics in ion temperature gradient (ITG) driven turbulence in a slab geometry.

We found that the entropy density with $n=2$ in the Hermite polynomial expansion in velocity space is important in convecting the entropy density, causing turbulent spreading.

The reminder of this paper is organized as follows. In section 2 , we briefly describe our physical model and derive a local entropy balance equation. Then, we investigate the entropy dynamics by a gyrokinetic Vlasov simulation in section 3. Finally, concluding remarks are given in section 4 . 


\section{Local Entropy Balance Equation}

To investigate global turbulent transport coupled with zonal flows in real space, we here employ a fourdimensional (4D) gyrokinetic model for electrostatic ITG turbulence in a slab geometry as a minimum kinetic model. The normalized basic equation system is then given as

$$
\begin{gathered}
\frac{\partial f}{\partial t}-\frac{\partial \Phi}{\partial y} \frac{\partial f}{\partial x}+\frac{\partial \Phi}{\partial x} \frac{\partial f}{\partial y}+v_{\|} \frac{\partial f}{\partial z}-\frac{\partial \Phi}{\partial z} \frac{\partial f}{\partial v_{\|}}=0, \\
-\left(\nabla^{2}+\frac{\rho_{\mathrm{ti}}^{2}}{\lambda_{\mathrm{Di}}^{2}} \nabla_{\perp}^{2}\right) \Phi+\frac{\rho_{\mathrm{ti}}^{2}}{\lambda_{\mathrm{De}}^{2}}\left(\Phi-<\Phi>_{y z}\right) \\
=\frac{\rho_{\mathrm{ti}}^{2}}{\lambda_{\mathrm{Di}}^{2}}\left(\int f d v_{\|}-1\right),
\end{gathered}
$$

where $f\left(t, x, y, z, v_{\|}\right)$and $\Phi(t, x, y, z)$ are the guiding center distribution function and electrostatic potential, respectively. The finite Larmor radius (FLR) effect is maintained only in the polarization density in the gyrokinetic Poisson equation [Eq. (2)], however, the essential features of nonlocal transport dynamics can be captured. The adiabatic response for electrons is assumed. Typical normalizations are applied,

$$
\begin{aligned}
& \left(x, y, z, v_{\|}, t, \Phi, f\right) \\
& \quad \rightarrow\left(\frac{x}{\rho_{i t}}, \frac{y}{\rho_{t i}}, \frac{z}{\rho_{t i}}, \frac{v_{\|}}{v_{t i}}, \frac{v_{t i} t}{\rho_{t i}}, \frac{e \Phi}{T_{0}}, \frac{v_{t i} f}{n_{0}}\right),
\end{aligned}
$$

where $v_{\mathrm{ti}}, \rho_{\mathrm{ti}}$ and $\lambda_{\mathrm{D} \sigma}(\sigma=$ ion, electron $)$ are the ion thermal velocity, ion Larmor radius and Debye length, respectively. In the present case, the density and electron temperature are assumed to be homogeneous, whereas the ion temperature has a profile in the $x$ (radial) direction.

The entropy balance equation is derived by multiplying Eq. (1) by $\delta f / f_{0}$ and integrating it over phase space,

$$
\begin{gathered}
\frac{\partial}{\partial t} \int \frac{\delta f^{2}}{2 f_{0}} d^{4} Z-\int \frac{\partial \Phi}{\partial y} \frac{\partial f_{0}}{\partial x} \frac{\delta f}{f_{0}} d^{4} Z-\int \frac{\partial \Phi}{\partial y} \frac{\partial f_{0}}{\partial x} \frac{\delta f^{2}}{2 f_{0}^{2}} d^{4} Z \\
+\int \frac{\partial \Phi}{\partial z} \frac{v_{\|}}{T_{i}} \delta f d^{4} Z+\int \frac{\partial \Phi}{\partial z} \frac{v_{\|}}{T_{i}} \frac{\delta f^{2}}{2 f_{0}} d^{4} Z=0,
\end{gathered}
$$

where $f_{0}$ and $\delta f=f-f_{0}$ denote the time- independent Maxwellian distribution function and its perturbed part, respectively. The terms on the left-hand side (LHS) of Eq. (4) represent the entropy production related to (a) phase space fluctuation, (b) heat flux, (c) higher-order term of (b), (d) wave-particle interactions such as Landau damping, and (e) higher-order term of (d), respectively. We define $\delta s \equiv \delta f^{2} / 2 f_{0}$, the integrand of (a), as perturbed entropy density. This entropy balance relationship among the terms has been investigated for an ITG turbulence in wavenumber space. It was found that (a) and (b) balance each other primarily in the transient phase near saturation and also in the quasi-steady state [6]. Note that the effect of zonal flows does not explicitly emerge in Eq. (4).

To study the effect of zonal flows, we derive the local entropy balance equation by retaining the $x$ dependence in the integration of Eq. (1),

$$
\begin{aligned}
\frac{\partial}{\partial t} \int \frac{\delta f^{2}}{2 f_{0}} d^{3} Z-\frac{\partial}{\partial x} \int \frac{\partial \Phi}{\partial y} \frac{\delta f^{2}}{2 f_{0}} d^{3} Z \\
-\int \frac{\partial \Phi}{\partial y} \frac{\partial f_{0}}{\partial x} \frac{\delta f}{f_{0}} d^{3} Z-\int \frac{\partial \Phi}{\partial y} \frac{\partial f_{0}}{\partial x} \frac{\delta f^{2}}{2 f_{0}^{2}} d^{3} Z \\
\quad+\int \frac{\partial \Phi}{\partial z} \frac{v_{\|}}{T_{i}} \delta f d^{3} Z+\int \frac{\partial \Phi}{\partial z} \frac{v_{\|}}{T_{i}} \frac{\delta f^{2}}{2 f_{0}} d^{3} Z=0,
\end{aligned}
$$

where $d^{3} Z=d y d z d v_{\|}$. In Eq. (5), the second term on the LHS is introduced, representing the convection of perturbed entropy density $\delta s$. Note that the first and second terms yield

$$
\frac{D\langle\delta s\rangle}{D t} \equiv \frac{\partial}{\partial t} \int \delta s d^{3} Z+\frac{\partial}{\partial x} \int\left(v_{E} \delta s\right) d^{3} Z,
$$

where $v_{\mathrm{E}}=-\partial_{y} \phi$. Another difference between Eqs. (5) and (4) is the third term, which is given by

$$
\begin{aligned}
& -\int \frac{\partial \Phi}{\partial y} \frac{\partial f_{0}}{\partial x} \frac{\delta f}{f_{0}} d^{3} Z \\
& =\frac{1}{2 L_{\mathrm{T}}} \int \frac{\partial \Phi}{\partial y} v_{\|}^{2} \delta f d^{3} Z-\frac{1}{2 L_{\mathrm{T}}} \int \frac{\partial \Phi}{\partial y} \delta f d^{3} Z,
\end{aligned}
$$

where $L_{\mathrm{T}}(x)=\partial_{x} T_{\mathrm{i}}(x) / T_{\mathrm{i}}(x)$. The first term on the right hand side (RHS) of Eq. (7) denotes the entropy density production related to local heat flux. The second term corresponds to the vorticity flow, which is found from Eq. (2) under the assumption that the Debye shielding effect is negligibly small,

$$
\int \frac{\partial \Phi}{\partial y} \delta f d^{3} Z=\int \frac{\partial \Phi}{\partial y} \nabla_{\perp}^{2} \Phi d y d z
$$

Thus, the RHS of Eq. (8) is found to be directly related to zonal flow production from the Hasegawa-Mima equation integrated in $(y, z)$ space:

$$
\iint \frac{\partial}{\partial t}\left(\frac{\partial \Phi}{\partial x}\right) d y d z-\iint \frac{\partial \Phi}{\partial y} \nabla_{\perp}^{2} \Phi d y d z=0 .
$$

The fourth, fifth and sixth terms in Eq. (5), which correspond to (c), (d) and (e), respectively, are found to be relatively small from the driftkinetic ordering. Therefore, the local entropy balance is established by the entropy density production related to phase space fluctuation, its convection, the zonal flow and the local heat flux.

\section{Entropy Dynamics in ITG Turbu- lence}

Here, we investigate the local entropy dynamics due to ITG turbulence using our global 4D (3D in real space and 1D in velocity space) gyrokinetic full-f Vlasov simulation based on the IDO-CF scheme [7]. We consider a shear-less slab geometry with a system size given by $L_{x}=2 L_{y}=32$, $L_{z}=8000$ in real space and $L_{v}=10$ in velocity space. The 

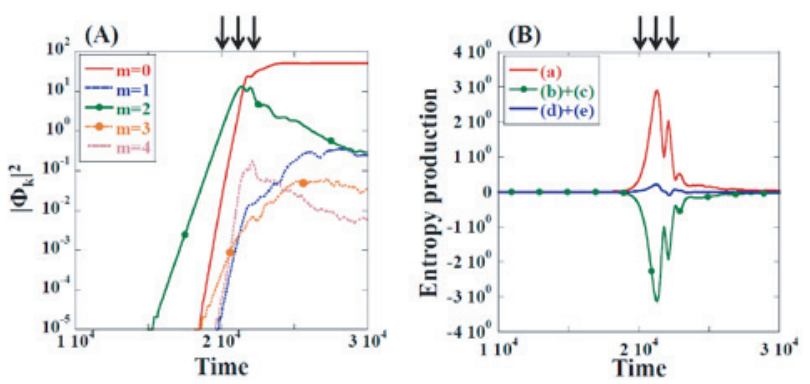

Fig. 1 Time evolution of (A) electrostatic potential with $m=$ $0,1,2,3,4$ and (B) each term in the entropy balance equation [Eq. (4)].

boundary condition is periodic, and the grid number is typically chosen as $\left(N_{x}, N_{y}, N_{z}, N_{v_{\|}}\right)=(256,64,32,128)$. The temperature profile is given by

$$
T_{\mathrm{i}}(x)=1-\frac{L_{x}}{2 \pi L_{\mathrm{T} 0}} \cos \left(\frac{2 \pi}{L_{x}} x\right),
$$

with $L_{\mathrm{T} 0}=37$. Note that there is no magnetic shear, so the ITG instability is excited in the radial region where the temperature gradient is steep.

Figure 1 (A) shows the time evolution of the electrostatic potential with different poloidal mode numbers. Zonal flows are found to play an important role in the saturation of ITG mode and the subsequent suppression of heat transport. Figure 1(B) shows the time evolution of each term in the entropy balance equation [Eq. (4)]. The entropy production due to phase space fluctuation (a) balances the heat flux (b) + (c) in the saturation phase and these values tend to zero because of quasi-linear flattening and zonal flow stabilization. The contribution of the wave-particle interactions (d) + (e) is found to be negligibly small. Note that the resolution in velocity space is limited by $\Delta v_{\|}=10 / 128$, so fine-scale structure smaller than this value is barely reproduced. This is found to happen at around $t=2.5 \times 10^{4}$ from the spectral analysis of the distribution function using the Hermite polynomial expansion, which is also used in Fig. 2.

Here, we focus on the local entropy dynamics at (1) $t=2.0 \times 10^{4},(2) t=2.1 \times 10^{4}$ and (3) $t=2.2 \times 10^{4}$ (marked by arrows in Fig. 1), where the turbulent state changes significantly. Note that the Hermite polynomial expansion converges at these time scales.

The spatial profile of each term in Eq. (5) is illustrated in the LHS column (A) in Fig. 2, i.e., the entropy density production related to the phase space fluctuation (EP), its convection (EC), the zonal flow (ZF) and the local heat flux (HF), respectively. The temperature profile is also illustrated. The higher-order terms, i.e., the fourth, fifth and sixth terms in Eq. (5), are numerically checked and found to be smaller by about one order of magnitude, as expected from the ordering (therefore, they are not shown in Fig. 2). The relative numerical error of the local entropy balance at each grid point is less than $1.7 \times 10^{-2}$ at $t=2.2 \times 10^{4}$.
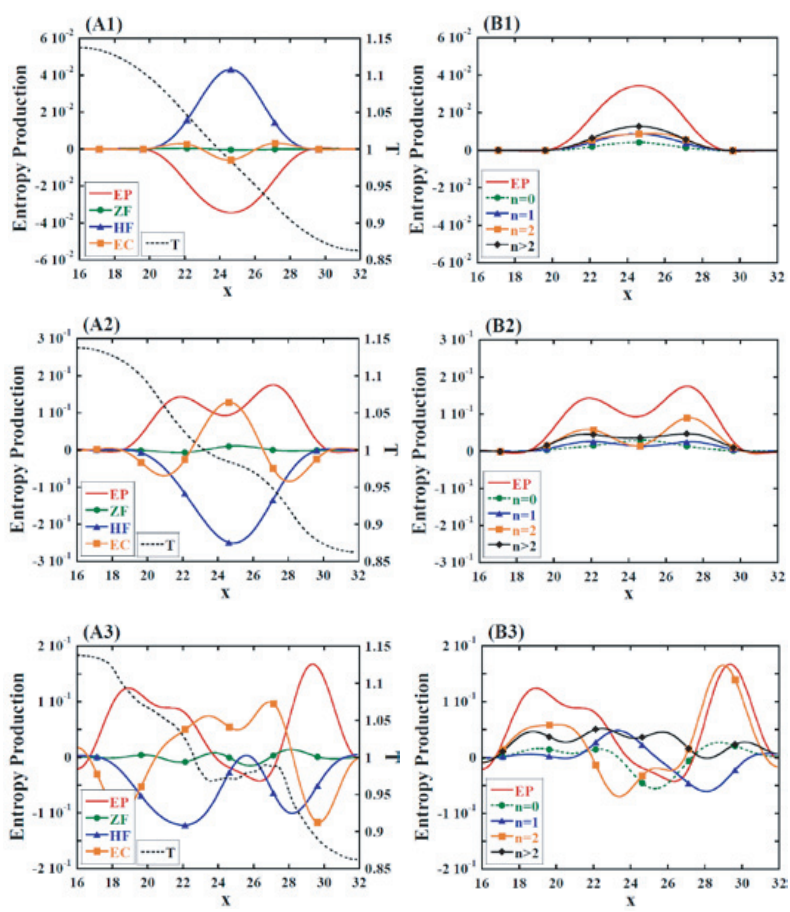

Fig. 2 (A) Spatial profiles of the entropy density production related to phase space fluctuation (EP), its convection (EC), zonal flow (ZF) and local heat flux (HF) at (1) $t=2.0 \times 10^{4}$, (2) $t=2.1 \times 10^{4}$ and (3) $t=2.2 \times 10^{4}$. Temperature profile is also shown. (B) Spatial profiles of the entropy density production (EP) for $n=0,1,2$ and $n>2$ with respect to the Hermite polynomial expansion.

To classify the role of perturbed entropy dynamics, we decompose the entropy density production (EP) by means of the Hermite polynomial expansion [8]. We show the spatial profile of EP for $n=0$ (density fluctuation), $n=1$ (momentum fluctuation), $n=2$ (temperature fluctuation) and the sum of the orders higher than $n=3$ with respect to the Hermite polynomial expansion in the RHS column (B).

In Fig. 2 (A1), which is the phase near the saturation, the local heat flux (HF) is induced due to the excitation of ITG mode where the temperature gradient is steep. Note that HF causes temperature relaxation and then flattening, as seen in Fig. 2 (A2). At this stage, a significant portion of the entropy density is found to be convected to both outer regions (see the EC profile). Then, the flattening of the temperature evolves further, leading to the propagation of fronts accompanied by the coupling between the entropy density production (EP) and convection (EC), as seen in Fig. 2 (A3). Note here that the effect of zonal flow (ZF) is found to be weak. This suggests that the zonal flow provides a small contribution in the units of entropy, whereas it overcomes the turbulent fluctuation in the unit of energy, as shown in Fig. 1 (A).

As seen in Fig. 2 (B1), the components with $n=1$ and $n=2$ in the Hermite polynomial expansion are found to be primary contributors to the entropy production, whereas 
the component with $n=0$, which is related to the zonal flow, contributes relatively less. Interestingly, as seen in Figs. 2 (B2) and (B3), the component with $n=2$ (temperature fluctuation) is important in convecting the entropy density to the outer regions. On the other hand, the zonal flow stays in a linearly unstable region and does not contribute to the convection. As a result, the front dynamics is dominated by the convection of the perturbed entropy with $n=2$.

\section{Conclusion}

We derived an entropy balance equation that maintains the dynamics in the radial direction, and can describe the spatio-temporal dynamics not only of the entropy density production related to zonal flow and local heat flux, but also of entropy density convection. Based on a 4D gyrokinetic Vlasov simulation in a slab geometry, we investigated the entropy dynamics in ITG turbulence.

In the phase where the turbulent state changes significantly, the entropy density production related to the heat flux balances not only that due to the phase space fluctuation, but also its convection, leading to front propagation. On the other hand, the contribution of zonal flow to entropy production is observed to be small even in the saturation phase, where the zonal flow energy is sufficiently large. This suggests that plasma dominated by large scale zonal flows corresponds to a lower entropy state.

From spectral analyses through the Hermite poly- nomial expansion, we found that temperature fluctuation is important in convecting the perturbed entropy density, causing turbulent spreading, whereas the zonal flow stays in a linearly unstable region and does not contribute to convection. This can be understood as indicating that zonal flow is low and/or zero frequency so that it hardly evolves in the radial direction. Front propagation is thus established primarily by the balance among the entropy density production related to phase space fluctuation with $n=2$, its convection and the local heat flux.

The present local entropy balance equation will be extended to an electron temperature gradient (ETG) driven turbulence system and also to a toroidal geometry.

[1] P. H. Diamond, S.-I. Itoh, K. Itoh and T. S. Hahm, Plasma Phys. Control Fusion 47, R35 (2005).

[2] Y. Kishimoto, T. Tajima, W. Horton, M. J. LeBrun and J. Y. Kim, Phys. Plasmas 3, 1289 (1996).

[3] T. S. Hahm, P. H. Diamond, Z. Lin, K. Itoh and S.-I. Itoh, Plasma Phys. Control. Fusion 46, A323 (2004).

[4] T. Matsumoto, J. Q. Li and Y. Kishimoto, Nucl. Fusion 47, 880 (2007).

[5] J. Anderson and E.-J. Kim, Nucl. Fusion 49, 075027 (2009).

[6] T.-H. Watanabe and H. Sugama, Phys. Plasmas 9, 3569 (2002).

[7] K. Imadera, Y. Kishimoto, D. Saito, J. Q. Li and T. Utsumi, J. Comput. Phys. 228, 8919 (2009).

[8] T.-H. Watanabe and H. Sugama, Phys. Plasmas 11, 1476 (2004). 